OPEN ACCESS

Edited by:

Hanting Zhang,

West Virginia University,

United States

Reviewed by:

Umesh Gangishetti,

Emory University, United States

Benson Wui-Man Lau,

Hong Kong Polytechnic University,

Hong Kong

Tao Yang.

University of Michigan, United States

*Correspondence:

Tatia M. C. Lee

tmclee@hku.hk

Received: 04 October 2016 Accepted: 09 May 2017

Published: 24 May 2017

Citation:

Lau WKW, Leung MK, Law ACK and Lee TMC (2017) Moderating Effects of Cortisol on Neural-Cognitive

Association in Cognitively Normal Elderly Subjects.

Front. Aging Neurosci. 9:163. doi: 10.3389/fnagi.2017.00163

\section{Moderating Effects of Cortisol on Neural-Cognitive Association in Cognitively Normal Elderly Subjects}

\author{
Way K. W. Lau 1,2, Mei Kei Leung ${ }^{2,3}$, Andrew C. K. Law ${ }^{1,4}$ and Tatia M. C. Lee $2,3,4,5 *$ \\ ${ }^{1}$ Neural Dysfunction Research Laboratory, Department of Psychiatry, The University of Hong Kong, Hong Kong, Hong Kong, \\ ${ }^{2}$ Laboratory of Cognitive Affective Neuroscience, The University of Hong Kong, Hong Kong, Hong Kong, ${ }^{3}$ Laboratory \\ of Neuropsychology, The University of Hong Kong, Hong Kong, Hong Kong, ${ }^{4}$ The State Key Laboratory of Brain and

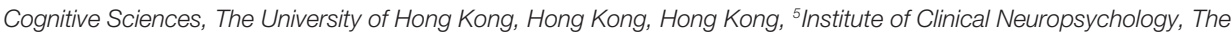 \\ University of Hong Kong, Hong Kong, Hong Kong
}

Cortisol homeostasis is important for healthy brain and cognitive aging. The aim of the current study is to investigate the role of serum cortisol levels in the relationship between regional brain volumes and cognitive processing speed in a group of cognitively normal elderly subjects. Forty-one healthy elderly participants were from a parallel longitudinal study. The reported data in this study reflects baseline measurements. Whole-brain anatomical scanning was performed using a 3.0 Tesla Philips Medical Systems Achieva scanner. Cognitive processing speed was assessed by the digit-symbol and symbol search tests, from the Chinese version of the Wechsler Adult Intelligence Scale-third edition (WAIS-III). Serum cortisol levels (sampled in the late morning) were measured by ELISA kits. Whole-brain regression analysis revealed that serum cortisol levels positively predicted the white matter volumes (WMV) of the right thalamus, the gray matter volumes (GMV) of the left thalamus and right cerebellar tonsil, and negatively predicted the WMV and GMV of the left middle temporal gyrus (MTG) in 41 healthy elderly participants. Furthermore, serum cortisol significantly moderated the relationship between the GMV of the left MTG and processing speed, as well as the GMV of the left thalamus and processing speed. This study provided the first piece of evidence supporting serum cortisol levels in moderating the relationship between regional brain volumes and processing speed in healthy elderly subjects. This observation enriches our understanding of the role of cortisol in brain morphology and cognitive functioning.

Keywords: cortisol, cognitive processing speed, brain volumes, moderation, neuroimaging

\section{INTRODUCTION}

Cortisol homeostasis is important for healthy cognitive aging. Dysregulation of the hypothalamus pituitary adrenal (HPA) axis, characterized by chronically elevated basal cortisol levels, is often associated with accelerated aging (Prenderville et al., 2015) and declined cognitive functioning in the elderly (Lupien et al., 2007, 2009; Comijs et al., 2010). Understanding the role of cortisol on neuro-cognitive functions in non-clinical elderly population would bring insight into the prevention of the development of neuro-cognitive disorders, e.g., dementia. Furthermore, it could also provide the scientific basis for stress reduction programs that improved cognitive functions in healthy aging adults (e.g., Galvin et al., 2006). 
The role of endogenous cortisol on cognitive function in healthy elderly subjects is relatively less studied. Thus far, only a few recent studies have demonstrated the association between cortisol and cognitive functions in healthy elderly subjects. For instance, Geerlings et al. (2015) observed that the morning cortisol levels measured 45 min after awakening were positively associated with cognitive processing speed and executive function, whereas the evening cortisol levels, sampled prior to sleep, were negatively associated with memory, cognitive processing speed, and executive function in healthy elderly subjects. Furthermore, low morning to evening cortisol ratio was shown to be associated with cognitive impairment in elderly men (Johar et al., 2015). In addition, Evans et al. (2012) also reported that executive function was positively linked to cortisol awakening response magnitude in a population of healthy older adults. These studies highlight the role of morning cortisol levels in cognitive function in elderly people. In fact, cortisol release is normally elevated in the morning to enhance energy, which has been reported to be associated with increased anticipatory states in 70 middle-class mothers (Adam and Gunnar, 2001), supporting the role of cortisol in facilitating cognitive performance. Nevertheless, there are also studies reporting no associations between diurnal cortisol levels, sampled at waking and $10 \mathrm{pm}$, and cognitive functions (e.g., Cox et al., 2015).

Cortisol has also been reported to be associated with changes in regional brain volumes. For instance, brain atrophy in bilateral hippocampal and cerebellar gray matter (GM) expressing high levels of glucocorticoid receptors (de Kloet et al., 2005; Hawrylycz et al., 2012) was observed in patients with uncontrolled Cushing's disease, a state with high cortisol levels in the blood (Burkhardt et al., 2015). A recent meta-analysis showed that the damaging effect of high cortisol levels in Cushing's syndrome on the human brain was irreversible (Andela et al., 2015). In healthy subjects, a brief hydrocortisone challenge was shown to significantly reduce hippocampal volume in a group of healthy adults (Brown et al., 2015). In addition, a recent study demonstrated a positive association between the morning cortisol levels and white matter volume (WMV) in the temporal, parietal and occipital lobes in a large sample of elderly subjects without dementia (Geerlings et al., 2015). In contrast, the morning plasma cortisol levels (at $9 \mathrm{am}$ ) have been reported to have no associations with age-related brain atrophy in a group of healthy elderly men (MacLullich et al., 2005), which may be due to the different sampling time.

There has been an increase in the number of studies reporting the relationship between cortisol, brain volumes and cognitive functions. The interaction between cortisol and its related regional brain volumes on cognitive functions is, however, less understood. In the present study, we aim to investigate the role of serum cortisol levels in the relationship between the regional brain volumes and cognitive status using moderation analyses in a group of non-depressed, cognitively normal elderly subjects. The cognitive processing speed was used to reflect the general state of cognitive functioning because it is an index sensitive to the general cognitive status (Salthouse, 1996). We hypothesized that serum cortisol significantly interacted with the regional brain volumes to predict processing speed outcomes. The findings from this study provided the first piece of evidence on the role of cortisol levels in neural-cognitive associations, which is important for monitoring the brain and cognitive aging.

\section{MATERIALS AND METHODS}

\section{Participants and Design}

This study was approved by the Institutional Review Board of The University of Hong Kong and the Hospital Authority. All subjects gave written informed consent in accordance with the Declaration of Helsinki. Inclusion criteria were 60 years old or above, fluent in Cantonese, education level of primary one or above, normal or corrected-to-normal hearing and vision, righthandedness, scored 8 or below in the 15-item version of the Geriatric Depression Scale (GDS-15) and scored 26 or above in the Montreal Cognitive Assessment, Hong Kong Version (HKMoCA, Wong et al., 2009). Subjects who scored 8 or below in GDS-15 were defined as normal or non-depressed (Boey and Chiu, 1998). A cutoff at 26 in HK-MoCA (after adjusted for the years of education) was chosen so as to be comparable with other international studies (Nasreddine et al., 2005). Subjects who scored 26 or above in HK-MoCA were defined as cognitively normal. Exclusion criteria were magnetic resonance imaging (MRI) incompatibility, history of brain injuries, neurological or psychiatric disease, endocrine disorders and current engagement in any psychotherapy or pharmacotherapy that may affect the functioning of autonomic and/or central nervous systems. Healthy elderly participants were from a parallel longitudinal study on meditation/relaxation training (Shao et al., 2016). Forty-one subjects who fitted in the abovementioned criteria were included in the current study. Serum cortisol sampling, assessments on cognitive processing speed and structural MRI were conducted within 3 weeks before the trainings, whereas cognitive assessments and structural MRI were conducted on the same day. The reported data in this study reflects baseline measurements.

\section{Processing Speed Index (PSI)}

Cognitive processing speed was assessed by two subtests, namely, the digit-symbol and symbol search test, from the Chinese version of the Wechsler Adult Intelligence Scale-third edition (WAIS-III; Wechsler et al., 2002). In the digit-symbol test, different symbols were paired with a set of numbers. Participants were asked to write or draw the symbols that correspond to particular numbers. In the symbol search test, participants were asked to identify the symbols that appeared repeatedly. The cumulative raw scores of the two subtests were summed to represent Processing speed index (PSI). Higher PSI score means higher processing speed.

\section{Structural MRI Data Acquisition and Processing}

Whole-brain anatomical scanning was performed using a 3.0 Tesla Philips Medical Systems Achieva scanner. A three- 
dimensional, T1-weighted, magnetization-prepared rapidacquisition gradient-echo (MP-RAGE) sequence was used to acquire high-resolution anatomical images (164 contiguous sagittal slices, $1-\mathrm{mm}$ thick, $\mathrm{TR}=7 \mathrm{~ms}, \mathrm{TE}=3.2 \mathrm{~ms}$, flip angle $=8^{\circ}, \mathrm{FOV}=164 \mathrm{~mm}$, matrix $=256 \times 240 \mathrm{~mm}$, voxel size $\left.=1 \mathrm{~mm}^{3}\right)$. The T1-images were processed using the VBM8 toolbox (Christian Gaser ${ }^{1}$ ). The default settings were used, unless otherwise specified. High-dimensional Dartel normalization approach was used (Ashburner, 2007). Modulated GM/white matter segments were generated by multiplying them with the nonlinear components derived from the normalization matrix instead of the linear components, in order to preserve the actual GM/white matter values locally and account for individual differences in global brain size. Finally, smoothing with an 8-mm full-width half-maximum (FWHM) Gaussian kernel was performed on the normalized modulated images. Serum cortisol levels were used to predict gray matter volumes (GMV) and WMV in the whole-brain. Clusters were considered significant at the combined voxel-extent threshold of an uncorrected voxel level of $p<0.001$ and cluster extent $\geq 160$ voxels, as determined by AlphaSim to be equivalent to $p<0.05$, corrected for multiple comparisons. Average GMV/WMV were extracted from the significant cluster(s) using the REX toolbox (Susan Whitfield-Gabrieli ${ }^{2}$ ) for further moderation analyses.

\section{Blood Sampling}

Blood sampling was conducted in the late morning to minimize the diurnal effect of cortisol (Touitou and Haus, 2000). The average blood sampling time was 10:52 am (standard deviation $(\mathrm{SD})=45 \mathrm{~min})$. Peripheral blood $(3 \mathrm{ml})$ was collected in vacutainers for each participant. Samples were allowed to clot for $30 \mathrm{~min}$ at room temperature before centrifugation at $1000 \times g$ for $15 \mathrm{~min}$ at room temperature. The upper serum layer was collected and stored at $-80^{\circ} \mathrm{C}$ until further analysis.

\section{Serum Cortisol Levels}

Serum cortisol levels were measured using a commercially available enzyme-linked immunosorbent assay (ELISA) kit from Enzo Life Sciences (Farmingdale, NY, USA) in duplicate per subject, according to the manufacturer's instructions. The detection range of the kit was $0.156-10 \mathrm{ng} / \mathrm{ml}$. The average intraassay coefficient of variation (CV) and inter-assay CV were $5.03 \%$ and $4.74 \%$, respectively.

\section{Statistical Analysis}

Normality of any continuous data was examined using the Kolmogorov-Smirnov test. Serum cortisol levels were transformed by natural logarithm and the transformed values were used for relevant parametric analyses. $P$-value $<0.05$ is considered statistically significant.

Moderation analyses were performed using model 1 in the PROCESS macro for SPSS developed by Hayes (2013).

\footnotetext{
${ }^{1}$ http://dbm.neuro.uni-jena.de/vbm/download/

${ }^{2}$ http://web.mit.edu/swg/software.htm
}

Briefly, the extracted brain volumes (predictors) and cortisol levels (moderators) were mean-centered. The mean-centered values together with their interaction term were entered in the linear regression model to predict processing speed indices. The effects of age, years of education and gender were adjusted (for the correlation matrix between covariates and variables of interest, please see Supplementary Table S1). The PROCESS macro is based on ordinary least squares regression and adopts a nonparametric bootstrapping procedure (1000 bootstrapped samples in this study), which gives rise to a bias-corrected confidence interval (CI) for effect size inference (Shrout and Bolger, 2002). The presence of a significant effect is denoted if zero is not included by the upper and lower bound of $95 \% \mathrm{CI}$ (Preacher and Hayes, 2008).

\section{RESULTS}

\section{Demographic Data}

We included 41 non-depressed, cognitively normal elderly men and women (age range 60-68 years old). The mean and standard deviation of serum cortisol levels were $32.34 \pm 46.50 \mathrm{ng} / \mathrm{mL}$ (range $=7.95-305.77 \mathrm{ng} / \mathrm{mL}$ ) in our samples, which is similar to the plasma cortisol levels reported in 95 healthy elderly men at 9 am (range $=38.48-329.10 \mathrm{ng} / \mathrm{mL}$, age range $65-70$ years old, MacLullich et al., 2005), and is much lower than the levels in Cushing's disease patients (range $=147.05-829.31 \mathrm{ng} / \mathrm{mL}$, Burkhardt et al., 2015). Details of the demographic data are reported in Table 1.

\section{Association between Serum Cortisol Levels and Regional Brain Volumes}

Whole-brain regression analysis revealed that serum cortisol levels positively predicted the WMV of the right thalamus, and negatively predicted the WMV of the left middle temporal gyrus (MTG). In addition, serum cortisol levels also positively predicted the GMV of the left thalamus and right cerebellar tonsil, and negatively predicted the GMV of the left MTG (Figure 1 and Table 2). The brain volumes of these regions were extracted to study the moderating role of serum cortisol levels in the association between processing speed and the brain volumes.

\begin{tabular}{lc}
\hline TABLE 1 | Demographic data of the enrolled elderly subjects $(\mathbf{N}=\mathbf{4 1})$. \\
\hline Variable & $\begin{array}{l}\text { Mean (Standard } \\
\text { Deviation) }\end{array}$ \\
\hline Age (years) & $64.71(2.35)$ \\
Gender & \\
$\quad$ Male & 15 \\
Female & 26 \\
Education (years) & $13.12(3.77)$ \\
Serum cortisol levels (ng/mL) & $32.34(46.50)$ \\
Montreal Cognitive Assessment (MoCA) score & $28.10(1.14)$ \\
15-item version of Geriatric Depression Scale (GDS-15) score & $2.90(2.34)$ \\
Processing speed index (PSI) & $92.17(18.02)$ \\
$\quad$ Digit symbol & $63.22(14.57)$ \\
$\quad$ Symbol search & $28.95(5.14)$
\end{tabular}




\section{A Thalamus}

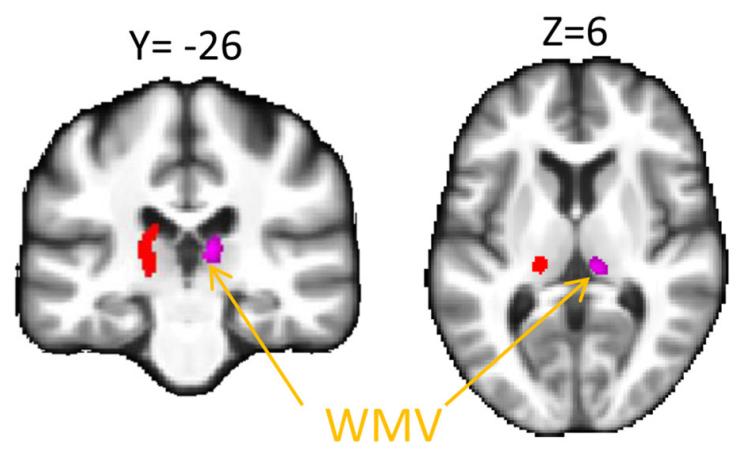

\section{B Cerebellar tonsil}
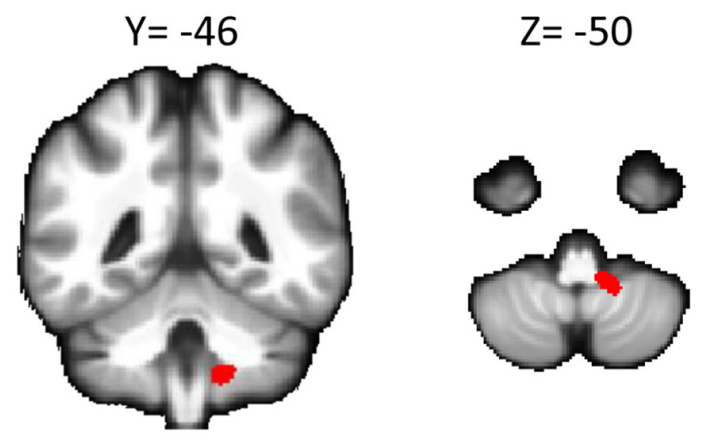

C MTG

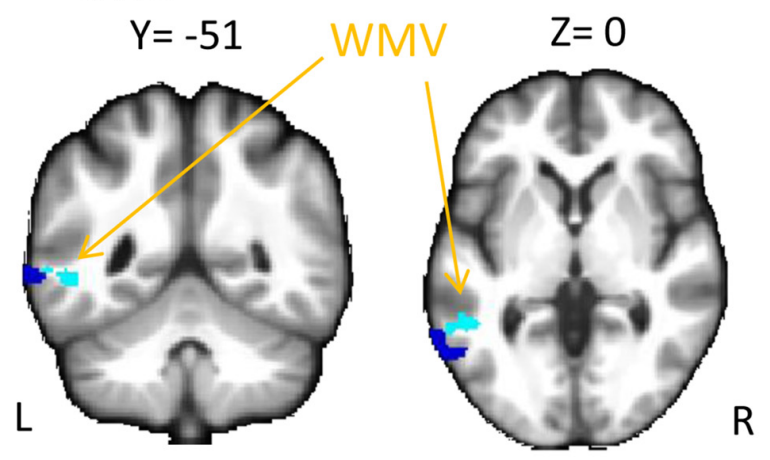

FIGURE 1 | Association between serum cortisol levels and gray matter volume (GMV) and white matter volume (WMV). Serum cortisol levels (transformed by natural logarithm) positively correlated with (A) GMV of the left thalamus (red clusters) and WMV of the right thalamus (violet clusters with arrows) and (B) GMV of the cerebellar tonsil (red clusters), and negatively correlated with (C) GMV (blue clusters) and WMV (light blue clusters with arrows) of the middle temporal gyrus (MTG). L, left; R, right.

\section{Moderating Role of Serum Cortisol in Neural-PSI Association}

\section{WMV of the Right Thalamus}

The interaction effect between serum cortisol and WMV of the right thalamus was not significant $(t=-1.917, p=0.064)$ after adjusted for the effect of age, years of education and gender.
Neither cortisol nor WMV of the right thalamus had a significant effect on PSI $(p>0.05)$.

\section{WMV of the Left MTG}

The interaction effect between serum cortisol and WMV of the left MTG was not significant ( $t=0.698, p=0.490)$ after adjusted for the effect of age, years of education and gender. Neither cortisol nor WMV of the left MTG had a significant effect on PSI $(p>0.05)$.

\section{GMV of the Right Cerebellar Tonsil}

The interaction effect between serum cortisol and the GMV of the right cerebellar tonsil was not significant $(t=-1.608$, $p=0.117$ ) after adjusted for the effect of age, years of education and gender. Neither cortisol nor the GMV of the right cerebellar tonsil had a significant effect on PSI $(p>0.05)$.

\section{GMV of the Left MTG}

The overall regression model in predicting PSI using serum cortisol levels, the GMV of the left MTG and their interaction term as independent variables was significant after controlled for the effects of age, years of education and gender $\left(F_{(6,34)}=4.100\right.$, $p=0.0034$ ), which accounted for $42.00 \%$ of variance in explaining PSI. The interaction term of serum cortisol levels and the GMV of the left MTG was a significant predictor of PSI (unstandardized coefficient $=118.430$, standard error $=57.875, p=0.0485$ ) . This indicated that serum cortisol levels moderated the relation between the GMV of the left MTG and PSI. Increased levels of serum cortisol reversed the effect of the GMV of the left MTG on PSI scores (Figure 2A). Neither serum cortisol nor the GMV of the left MTG had a significant effect on PSI $(p>0.05)$, suggesting a complete moderation. In the sub-scale analysis, serum cortisol levels were found to significantly moderate the relationship between the GMV of the left MTG and digit symbol scores, but not symbol search scores (Table 3).

\section{GMV of the Left Thalamus}

The overall regression model in predicting PSI using serum cortisol levels, the GMV of the left thalamus and their interaction term as independent variables was significant after controlled for the effects of age, years of education and gender $\left(F_{(6,34)}=4.946, p=0.0010\right)$, which accounted for $46.61 \%$ of variance in explaining PSI. The GMV of the left thalamus, but not serum cortisol, had a significant effect on PSI (unstandardized coefficient $=167.484$, standard error $=81.508$, $p=0.0476$ ). The interaction term of serum cortisol levels and the GMV of the left thalamus was a significant predictor of PSI (unstandardized coefficient $=-204.201$, standard error $=93.475$, $p=0.0359)$, indicating that serum cortisol levels also moderated the relationship between the GMV of the left thalamus and PSI. Increased levels of serum cortisol reversed the effect of the GMV of the left thalamus on PSI scores (Figure 2B). In sub-scale analysis, serum cortisol levels were found to significantly moderate the relationship between the GMV of the left thalamus and symbol search scores, but not digit symbol scores (Table 3). 
TABLE 2 | Regression with whole-brain GMV and WMV predicted by serum cortisol level.

\begin{tabular}{|c|c|c|c|c|c|c|c|}
\hline & \multirow[t]{2}{*}{ Association with serum cortisol level } & \multirow[t]{2}{*}{ Brain region } & \multicolumn{3}{|c|}{ Peak coordinates in MNI space } & \multirow[t]{2}{*}{$t$ - value } & \multirow[t]{2}{*}{ Cluster size } \\
\hline & & & $X$ & $Y$ & $Z$ & & \\
\hline \multirow[t]{4}{*}{ WMV } & Positive & Right thalamus & 10 & -25 & 9 & 4.90 & $201^{*}$ \\
\hline & Negative & Left middle temporal gyrus & -50 & -48 & -2 & 3.87 & $203^{*}$ \\
\hline & & & -58 & -46 & 3 & 3.83 & \\
\hline & & & -57 & -57 & -2 & 3.59 & \\
\hline \multirow[t]{6}{*}{ GMV } & Positive & Left thalamus & -15 & -27 & 16 & 4.48 & $241^{*}$ \\
\hline & & & -18 & -25 & 3 & 3.99 & \\
\hline & Positive & Right cerebellar tonsil & 12 & -43 & -51 & 4.54 & $186^{*}$ \\
\hline & Negative & Left middle temporal gyrus & -57 & -60 & 4 & 4.36 & $491^{*}$ \\
\hline & & & -68 & -54 & -3 & 3.75 & \\
\hline & & & -54 & -61 & 15 & 3.73 & \\
\hline
\end{tabular}

Note: GMV, Gray Matter Volume; MNI, Montreal Neurological Institute; WMV, White Matter Volume. *Corrected p < 0.05 as defined by uncorrected voxel-level threshold $p<0.001$ and cluster-level threshold $\geq 160$ voxels.

\section{DISCUSSION}

The current study applied moderation analyses to understand the role of serum cortisol levels in the relationship between regional brain volumes and cognitive processing speed. Our study emphasized on the moderating role of serum cortisol levels in the neural-cognitive association. We also demonstrated associations between serum cortisol levels and regional brain volumes, including both the GMV and WMV, which corroborates some of the previous observations from the literature. Our findings support the importance of cortisol homeostasis in monitoring morphological and cognitive changes seen in healthy elderly people.

In a previous study on a large sample of elderly subjects without dementia, the morning saliva cortisol levels (sampled 45 min after awakening) were shown to be positively associated with the WMV in different lobes of the brain as well as processing speed and executive function, but not with the GMV (Geerlings et al., 2015). In this study, using a whole brain voxel wise approach, we found that the late morning serum cortisol levels were positively associated with the WMV of the right thalamus, the GMV of the left thalamus and right cerebellar tonsil, and were negatively associated with the GMV and WMV of the left MTG (Figure 1, Table 2). The differences in the findings between Geerlings's and our study may be due to the use of average volume of bilateral brain regions in their study, which may render the investigation of the association between morning cortisol levels and GM less sensitive, especially if the effect is laterality-specific. Also, the difference in cortisol sampling time between the two studies could potentially affect the outcomes. The brain regions that we found to be correlated with serum cortisol levels express glucocorticoid receptors in humans (Sarrieau et al., 1988; Yu et al., 2002) and have been reported to be associated with cognitive functions. For example, higher high-gamma power in the left thalamus was associated with better processing speed among healthy elderly adults using magnetoencephalogram (Akimoto et al., 2014). Hypometabolism in the right cerebellar tonsil has been reported in illiterate elderly subjects with poorer cognitive functions compared to age- and gender-matched literate subjects (Kwon et al., 2012). The role of cerebellum in controlling incoming working memory information was also demonstrated in patients with cerebellar ischemic stroke (Baier et al., 2014). The positive associations between serum cortisol levels and these regions may imply a facilitating role of cortisol in cognitive functions via these brain regions. On the other hand, atrophy of the bilateral thalamus and left MTG were consistently found in patients with mild cognitive impairment or dementia (Yang et al., 2012a,b). The negative association between serum cortisol levels and regional brain volumes of the left MTG indicates a region-specific response to cortisol in the MTG, which is different from the cortisol response in the thalamus and cerebellum. This observation requires more future studies to confirm. Taken together, our findings suggest the close relationship between the morning serum cortisol levels and the WMV/GMV of brain regions that are important for cognitive functions in cognitively normal elderly people.

We further investigated the moderating role of serum cortisol levels in the neural-cognitive association that involved the abovementioned brain regions. From the moderation analyses, we found a significant moderating role of the serum cortisol levels in the relationship between the GMV of the left MTG and PSI. Increased cortisol levels changed the relationship between the GMV of the left MTG and PSI from negative to positive (Figure 2A), suggesting that cortisol facilitates the left MTG in performing the cognitive task. The MTG is associated with many different functions, such as language processing (Mirz et al., 1999), observation of motion (Rizzolatti et al., 1996), deductive reasoning (Goel et al., 1998) and dynamic facial expressions (Sato et al., 2012). Recently, different parts of the MTG have been reported to functionally and anatomically connect with other brain regions that are involved in memory retrieval (e.g., hippocampus) and executive controls (e.g., inferior frontal gyrus; $\mathrm{Xu}$ et al., 2015), which is believed to have substantial influence on processing speed (Cepeda et al., 2013). Interestingly, the moderating effect of serum cortisol levels was specific to the digit symbol sub-scales of PSI, of which the performance is significantly contributed by memory functions assessed by incidental learning and Wechsler Memory Scale III (immediate and delayed recall for visual and auditory domains; Joy et al., 2004). Our findings suggest 


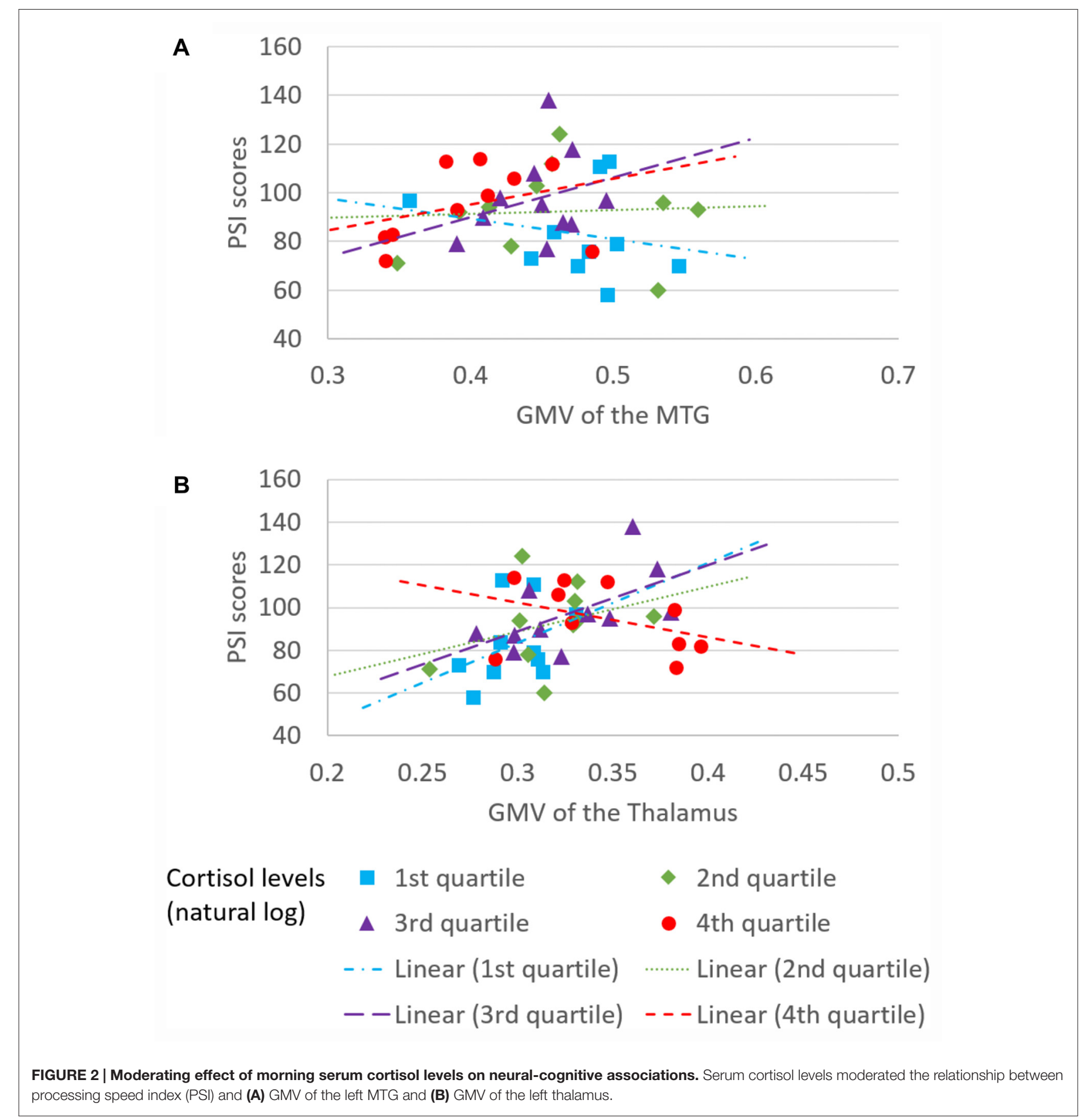

that the effect of the interaction between cortisol and the MTG on processing speed involves a memory component. Unfortunately, memory domains were not captured in the current study. Therefore, such speculation requires further studies to confirm.

We also found a significant moderating role of the serum cortisol levels in the association between the GMV of the left thalamus and the PSI. In contrast to the finding in the MTG, positive associations between the GMV of the left thalamus and PSI were observed in subjects with low to medium serum cortisol levels (from the 1 st to the $3 \mathrm{rd}$ quartile, Figure 2B), whereas a negative association between the GMV of the left thalamus and PSI was observed in subjects with high serum cortisol levels (the 4th quartile, Figure 2B). Furthermore, such moderating effect was specific to the other sub-scale of PSI-the symbol search task-that does not rely on memory as much as the digit symbol task. One speculation for this observation is that glucocorticoid 
TABLE 3 | Moderating role of serum cortisol in the association between the regional GMV and processing speed index (PSI) subscales.

\begin{tabular}{|c|c|c|c|c|}
\hline & Unstandardized coefficient & Standard error & $t$-value & $p$-value \\
\hline \multicolumn{5}{|l|}{ GMV of the left MTG } \\
\hline \multicolumn{5}{|l|}{$P S I$} \\
\hline Serum cortisol levels & 9.897 & 5.166 & 1.916 & 0.064 \\
\hline Interaction term & 118.430 & 57.875 & 2.046 & 0.049 \\
\hline \multicolumn{5}{|l|}{ Digit symbol } \\
\hline Serum cortisol levels & 6.500 & 4.302 & 1.511 & 0.140 \\
\hline \multicolumn{5}{|l|}{ Symbol search } \\
\hline Serum cortisol levels & 3.396 & 1.571 & 2.162 & 0.038 \\
\hline GMV of the left MTG & 14.442 & 17.176 & 0.841 & 0.406 \\
\hline Interaction term & 18.823 & 17.596 & 1.070 & 0.292 \\
\hline \multicolumn{5}{|l|}{ GMV of the left thalamus } \\
\hline Serum cortisol levels & 1.563 & 4.194 & 0.373 & 0.712 \\
\hline GMV of the left thalamus & 128.720 & 69.657 & 1.848 & 0.073 \\
\hline Interaction term & -139.901 & 79.884 & -1.751 & 0.089 \\
\hline \multicolumn{5}{|l|}{ Symbol search } \\
\hline Serum cortisol levels & 2.756 & 1.452 & 1.898 & 0.066 \\
\hline GMV of the left thalamus & 38.764 & 24.120 & 1.607 & 0.117 \\
\hline Interaction term & -64.300 & 27.661 & -2.325 & 0.026 \\
\hline
\end{tabular}

Note: GMV, Gray matter volume; PSI, Processing speed index. The effect of age, years of education and gender were adjusted. P-values < 0.05 were bold.

receptors are more densely expressed in the thalamus. Within the optimal range of cortisol, the bigger volume of the thalamus (plausibly with more glucocorticoid receptors) could bind more cortisol, which subsequently influences perceptual detection thresholds. This can then enhance focused attention on the perceived stimuli and excludes irrelevant stimuli (Fehm-Wolfsdorf and Nagel, 1996; Erickson et al., 2003), resulting in better processing speed. In contrast, excess cortisol can down-regulate glucocorticoid receptors in the brain (Sapolsky and McEwen, 1985), which reduces the regional brain responsiveness to cortisol stimulations, and hence, minimizes the effect of cortisol on processing speed. Specifically, the thalamic region that we found to be associated with cortisol levels was located approximately at the lateral thalamic nuclei that were functionally related to spatial attentional weighting, selective attentional control and visual perceptual processing speed (Kraft et al., 2015). We, therefore, speculate that the cortisol-thalamic interaction effect on cognitive processing speed may be mediated through visual attentional processing. This speculation requires more empirical studies to confirm. Consistent with the literature, our findings further corroborate the non-linear relationship between cortisol levels and cognitive function (for review see Belanoff et al., 2001) by interacting with the regional brain volumes. The differential interaction effects between serum cortisol levels, the MTG and the thalamus might be due to the regionalspecific reduction in glucocorticoid receptors in the brain during aging, which requires more empirical studies to confirm.

\section{LIMITATIONS}

There are several limitations in the current study. First, interpretations on results are limited by the current study design. Although we adopted moderation models, the causality among those markers of interest cannot be confirmed without a longitudinal design. Second, serum cortisol was sampled only once in the late morning $(\sim 11 \mathrm{am})$. The effect of cortisol awakening response and the diurnal effect of cortisol on the regional brain volumes and cognitive function cannot be deduced in the current study. Third, the absence of information on other domains of cognitive ability or other brain imaging modalities would limit our interpretations on the results. Furthermore, cautions should be taken when interpreting the results due to the limited sample size in the current study. Last, blood sampling was done beyond the morning period $(12-1 \mathrm{pm})$ in four subjects. Nonetheless, all significant findings maintained after these four subjects were removed in a sensitivity test, suggesting the effect of this small delay on our findings is minimal. Future studies that directly manipulate the level of cortisol with a greater sample size could provide a more direct impact of this stress marker on the brain and cognitive aging.

\section{CONCLUSION}

To conclude, our study provided the first piece of evidence to support the moderating effects of serum cortisol levels on the relationship between regional brain volumes and processing 
speed. Our findings provide insight into the role of cortisol in modulating brain morphology and cognitive functions in healthy elderly subjects.

\section{AUTHOR CONTRIBUTIONS}

TMCL and ACKL conceived and designed the study. TMCL supervised data acquisition. WKWL and MKL analyzed the data. WKWL interpreted the data and drafted the article. All authors revised the article critically for important intellectual content and have approved the final article.

\section{REFERENCES}

Adam, E. K., and Gunnar, M. R. (2001). Relationship functioning and home and work demands predict individual differences in diurnal cortisol patterns in women. Psychoneuroendocrinology 26, 189-208. doi: 10.1016/s03064530(00)00045-7

Akimoto, Y., Nozawa, T., Kanno, A., Ihara, M., Goto, T., Ogawa, T., et al. (2014). High-gamma activity in an attention network predicts individual differences in elderly adults' behavioral performance. Neuroimage 100, 290-300. doi: 10.1016/j.neuroimage.2014.06.037

Andela, C. D., van Haalen, F. M., Ragnarsson, O., Papakokkinou, E., Johannsson, G., Santos, A., et al. (2015). Mechanisms in endocrinology: cushing's syndrome causes irreversible effects on the human brain: a systematic review of structural and functional magnetic resonance imaging studies. Eur. J. Endocrinol. 173, R1-R14. doi: 10.1530/EJE-14-1101

Ashburner, J. (2007). A fast diffeomorphic image registration algorithm. Neuroimage 38, 95-113. doi: 10.1016/j.neuroimage.2007.07.007

Baier, B., Müller, N. G., and Dieterich, M. (2014). What part of the cerebellum contributes to a visuospatial working memory task? Ann. Neurol. 76, 754-757. doi: 10.1002/ana.24272

Belanoff, J. K., Gross, K., Yager, A., and Schatzberg, A. F. (2001). Corticosteroids and cognition. J. Psychiatr. Res. 35, 127-145. doi: 10.1016/s00223956(01)00018-8

Boey, K. M., and Chiu, H. F. K. (1998). Assessing psychological well-being of the old-old: a comparative study of GDS-15 and GHQ-12. Clin. Gerontol. 19, 65-75. doi: 10.1300/j018v19n01_06

Brown, E. S., Jeon-Slaughter, H., Lu, H., Jamadar, R., Issac, S., Shad, M., et al. (2015). Hippocampal volume in healthy controls given 3-day stress doses of hydrocortisone. Neuropsychopharmacology 40, 1216-1221. doi: 10.1038/npp. 2014.307

Burkhardt, T., Ludecke, D., Spies, L., Wittmann, L., Westphal, M., and Flitsch, J. (2015). Hippocampal and cerebellar atrophy in patients with Cushing's disease. Neurosurg. Focus 39:E5. doi: 10.3171/2015.8.FOCUS15324

Cepeda, N. J., Blackwell, K. A., and Munakata, Y. (2013). Speed isn't everything: complex processing speed measures mask individual differences and developmental changes in executive control. Dev. Sci. 16, 269-286. doi: 10.1111/desc.12024

Comijs, H. C., Gerritsen, L., Penninx, B. W., Bremmer, M. A., Deeg, D. J., and Geerlings, M. I. (2010). The association between serum cortisol and cognitive decline in older persons. Am. J. Geriatr. Psychiatry 18, 42-50. doi: 10.1097/JGP. 0b013e3181b970ae

Cox, S. R., MacPherson, S. E., Ferguson, K. J., Royle, N. A., Maniega, S. M., Hernández Mdel, C., et al. (2015). Does white matter structure or hippocampal volume mediate associations between cortisol and cognitive ageing? Psychoneuroendocrinology 62, 129-137. doi: 10.1016/j.psyneuen.2015. 08.005

de Kloet, E. R., Joëls, M., and Holsboer, F. (2005). Stress and the brain: from adaptation to disease. Nat. Rev. Neurosci. 6, 463-475. doi: 10.1038/nr n1683

Erickson, K., Drevets, W., and Schulkin, J. (2003). Glucocorticoid regulation of diverse cognitive functions in normal and pathological emotional states. Neurosci. Biobehav. Rev. 27, 233-246. doi: 10.1016/s0149-7634(03)00033-2

\section{FUNDING}

This work was supported by the General Research Fund of the Hong Kong Research Grant Council (HKU17613815).

\section{SUPPLEMENTARY MATERIAL}

The Supplementary Material for this article can be found online at: http://journal.frontiersin.org/article/10.3389/fnagi.2017.001 63/full\#supplementary-material

Evans, P., Hucklebridge, F., Loveday, C., and Clow, A. (2012). The cortisol awakening response is related to executive function in older age. Int. J. Psychophysiol. 84, 201-204. doi: 10.1016/j.ijpsycho.2012. 02.008

Fehm-Wolfsdorf, G., and Nagel, D. (1996). Differential effects of glucocorticoids on human auditory perception. Biol. Psychol. 42, 117-130. doi: 10.1016/03010511(95)05150-3

Galvin, J. A., Benson, H., Deckro, G. R., Fricchione, G. L., and Dusek, J. A. (2006). The relaxation response: reducing stress and improving cognition in healthy aging adults. Complement. Ther. Clin. Pract. 12, 186-191. doi: 10.1016/j.ctcp. 2006.02.004

Geerlings, M. I., Sigurdsson, S., Eiriksdottir, G., Garcia, M. E., Harris, T. B., Gudnason, V., et al. (2015). Salivary cortisol, brain volumes, and cognition in community-dwelling elderly without dementia. Neurology 85, 976-983. doi: 10.1212/WNL.0000000000001931

Goel, V., Gold, B., Kapur, S., and Houle, S. (1998). Neuroanatomical correlates of human reasoning. J. Cogn. Neurosci. 10, 293-302. doi: 10.1162/089892998562744

Hawrylycz, M. J., Lein, E. S., Guillozet-Bongaarts, A. L., Shen, E. H., Ng, L., Miller, J. A., et al. (2012). An anatomically comprehensive atlas of the adult human brain transcriptome. Nature 489, 391-399. doi: 10.1038/nature11405

Hayes, A. F. (2013). An Introduction to Mediation, Moderation and Conditional Process Analysis: A Regression-Based Approach. New York, NY: Guilford Press.

Johar, H., Emeny, R. T., Bidlingmaier, M., Lacruz, M. E., Reincke, M., Peters, A., et al. (2015). Lower morning to evening cortisol ratio is associated with cognitive impairment in men but not women: an analysis of 733 older subjects of the cross-sectional KORA-Age study. Psychoneuroendocrinology 51, 296-306. doi: 10.1016/j.psyneuen.2014.10.011

Joy, S., Kaplan, E., and Fein, D. (2004). Speed and memory in the WAIS-III digit symbol-coding subtest across the adult lifespan. Arch. Clin. Neuropsychol. 19, 759-767. doi: 10.1016/j.acn.2003.09.009

Kraft, A., Irlbacher, K., Finke, K., Kaufmann, C., Kehrer, S., Liebermann, D., et al. (2015). Dissociable spatial and non-spatial attentional deficits after circumscribed thalamic stroke. Cortex 64, 327-342. doi: 10.1016/j.cortex.2014. 12.005

Kwon, O. D., Cho, S. S., Seo, S. W., and Na, D. L. (2012). Effect of illiteracy on neuropsychological tests and glucose metabolism of brain in later life. J. Neuroimaging 22, 292-298. doi: 10.1111/j.1552-6569.2011.00618.x

Lupien, S. J., Maheu, F., Tu, M., Fiocco, A., and Schramek, T. E. (2007). The effects of stress and stress hormones on human cognition: implications for the field of brain and cognition. Brain Cogn. 65, 209-237. doi: 10.1016/j.bandc.2007.02. 007

Lupien, S. J., McEwen, B. S., Gunnar, M. R., and Heim, C. (2009). Effects of stress throughout the lifespan on the brain, behaviour and cognition. Nat. Rev. Neurosci. 10, 434-445. doi: 10.1038/nrn2639

MacLullich, A. M., Deary, I. J., Starr, J. M., Ferguson, K. J., Wardlaw, J. M., and Seckl, J. R. (2005). Plasma cortisol levels, brain volumes and cognition in healthy elderly men. Psychoneuroendocrinology 30, 505-515. doi: 10.1016/j. psyneuen.2004.12.005

Mirz, F., Ovesen, T., Ishizu, K., Johannsen, P., Madsen, S., Gjedde, A., et al. (1999). Stimulus-dependent central processing of auditory stimuli: a PET study. Scand. Audiol. 28, 161-169. doi: 10.1080/010503999424734 
Nasreddine, Z. S., Phillips, N. A., Bédirian, V., Charbonneau, S., Whitehead, V., Collin, I., et al. (2005). The montreal cognitive assessment, MoCA: a brief screening tool for mild cognitive impairment. J. Am. Geriatr. Soc. 53, 695-699. doi: 10.1111/j.1532-5415.2005.53221.x

Preacher, K. J., and Hayes, A. F. (2008). Asymptotic and resampling strategies for assessing and comparing indirect effects in multiple mediator models. Behav. Res. Methods 40, 879-891. doi: 10.3758/brm.40.3.879

Prenderville, J. A., Kennedy, P. J., Dinan, T. G., and Cryan, J. F. (2015). Adding fuel to the fire: the impact of stress on the ageing brain. Trends Neurosci. 38, 13-25. doi: 10.1016/j.tins.2014.11.001

Rizzolatti, G., Fadiga, L., Matelli, M., Bettinardi, V., Paulesu, E., Perani, D., et al. (1996). Localization of grasp representations in humans by PET: 1. Observation versus execution. Exp. Brain Res. 111, 246-252. doi: 10.1007/bf002 27301

Salthouse, T. A. (1996). The processing-speed theory of adult age differences in cognition. Psychol. Rev. 103, 403-428. doi: 10.1037/0033-295x.103. 3.403

Sapolsky, R. M., and McEwen, B. S. (1985). Down-regulation of neural corticosterone receptors by corticosterone and dexamethasone. Brain Res. 339, 161-165. doi: 10.1016/0006-8993(85)90638-9

Sarrieau, A., Dussaillant, M., Sapolsky, R. M., Aitken, D. H., Olivier, A., Lal, S., et al. (1988). Glucocorticoid binding sites in human temporal cortex. Brain Res. 442, 157-160. doi: 10.1016/0006-8993(88)91444-8

Sato, W., Toichi, M., Uono, S., and Kochiyama, T. (2012). Impaired social brain network for processing dynamic facial expressions in autism spectrum disorders. BMC Neurosci. 13:99. doi: 10.1186/1471-2202-13-99

Shao, R., Keuper, K., Geng, X., and Lee, T. M. (2016). Pons to posterior cingulate functional projections predict affective processing changes in the elderly following eight weeks of meditation training. EBioMedicine 10, 236-248. doi: 10.1016/j.ebiom.2016.06.018

Shrout, P. E., and Bolger, N. (2002). Mediation in experimental and nonexperimental studies: new procedures and recommendations. Psychol. Methods 7, 422-445. doi: 10.1037/1082-989x.7.4.422
Touitou, Y., and Haus, E. (2000). Alterations with aging of the endocrine and neuroendocrine circadian system in humans. Chronobiol. Int. 17, 369-390. doi: $10.1081 / \mathrm{cbi}-100101052$

Wechsler, D., Chen, Y. H., and Chen, X. Y. (2002). WAIS-III Chinese Version Technical Manual. San Antonio, TX: Psychological Corporation.

Wong, A., Xiong, Y. Y., Kwan, P. W., Chan, A. Y., Lam, W. W., Wang, K., et al. (2009). The validity, reliability and clinical utility of the hong kong montreal cognitive assessment (HK-MoCA) in patients with cerebral small vessel disease. Dement. Geriatr. Cogn. Disord. 28, 81-87. doi: 10.1159/000232589

Xu, J., Wang, J., Fan, L., Li, H., Zhang, W., Hu, Q., et al. (2015). Tractographybased parcellation of the human middle temporal gyrus. Sci. Rep. 5:18883. doi: $10.1038 /$ srep 18883

Yang, J., Pan, P., Song, W., Huang, R., Li, J., Chen, K., et al. (2012a). Voxelwise meta-analysis of gray matter anomalies in Alzheimer's disease and mild cognitive impairment using anatomic likelihood estimation. J. Neurol. Sci. 316, 21-29. doi: 10.1016/j.jns.2012.02.010

Yang, J., Pan, P., Song, W., and Shang, H. F. (2012b). Quantitative meta-analysis of gray matter abnormalities in semantic dementia. J. Alzheimers Dis. 31, 827-833. doi: 10.3233/JAD-2012-120736

Yu, L., Romero, D. G., Gomez-Sanchez, C. E., and Gomez-Sanchez, E. P. (2002). Steroidogenic enzyme gene expression in the human brain. Mol. Cell. Endocrinol. 190, 9-17. doi: 10.1016/s0303-7207(02)00041-2

Conflict of Interest Statement: The authors declare that the research was conducted in the absence of any commercial or financial relationships that could be construed as a potential conflict of interest.

Copyright $\odot 2017$ Lau, Leung, Law and Lee. This is an open-access article distributed under the terms of the Creative Commons Attribution License (CC BY). The use, distribution or reproduction in other forums is permitted, provided the original author(s) or licensor are credited and that the original publication in this journal is cited, in accordance with accepted academic practice. No use, distribution or reproduction is permitted which does not comply with these terms. 